\title{
Lesiones bucales características de la sífilis, encontradas en tres servicios de estomatología de Latinoamérica
}

\author{
Characteristic Oral Lesions of Syphilis Found \\ in Three Stomatology Services in Latin America
}

\author{
Martha Carmona, MSc. ${ }^{1}$, Iván Porto $\bowtie$, OD.${ }^{1}$, Yelisa Ríos, MSc. ${ }^{2}$, \\ Héctor Lanfranchi, MSc. ${ }^{3}$
}

\footnotetext{
${ }^{1}$ Universidad de Cartagena, Cartagena, Colombia

2 Universidad de Buenos Aires, Buenos Aires, Argentina

${ }^{3}$ Universidad de Buenos Aires, Buenos Aires, Argentina
}

Facultad de Odontología, Universidad de Cartagena, sede Zaragocilla, Carrera 50 n. ${ }^{2}$ 29-11. Correo electrónico: iportop@unicartagena.edu.co

Recibido: 3 de abril del 2017 Aprobado: 20 de mayo del 2017

Cómo citar este artículo: Carmona M, Porto I, Ríos Y, Lanfranchi H. Lesiones bucales características de la sífilis, encontradas en tres servicios de estomatología de Latinoamérica. Rev Nac Odontol. 2018;13(26):1-8. doi: http://dx.doi.org/10.16925/ od.v13i26.1788

\section{Resumen}

Introducción: la sífilis es una enfermedad considerada como antigua, que ha causado grandes estragos durante toda la historia de la humanidad, y a pesar de métodos de protección sexual y antibióticos para combatir esta infección, en la actualidad todavía su prevalencia es preocupante. El odontólogo juega un papel imprescindible en este campo, debido a que debe ser capacitado para detectar las manifestaciones de la enfermedad en cavidad bucal de una forma temprana, con lo que se evitaría su progresión.

Metodología: se realiza un estudio descriptivo de corte transversal, con veinte pacientes que consultaron tres servicios de estomatología de Suramérica; se les realiza examen estomatológico e historia clínica exhaustiva; se clasificaron las lesiones encontradas y se analizaron los demás datos recogidos. Resultados: la lesión prevalente en sífilis primaria fue la úlcera y solo se detectó un caso; acerca de la forma secundaria se detectaron diecinueve casos y se encontraron múltiples lesiones asociadas; por último, no se encontró ningún caso de sífilis terciaria.

Conclusiones: se considera fundamental la divulgación de estos resultados y mayores investigaciones en este campo, con el fin de educar y así evitar la progresión de la sífilis a una forma latente o terciaria que ponga en riesgo la vida de la población.

Palabras clave: enfermedades de transmisión sexual, neoplasias de la boca, serodiagnóstico de la sifilis, sífilis. 


\title{
Characteristic Oral Lesions of Syphilis Found in Three Stomatology Services in Latin America
}

\begin{abstract}
Introduction: Syphilis is an ancient disease that has caused great damage throughout the history of humanity and, despite sexual protection methods and antibiotics to combat this infection, its prevalence is still alarming today. The dentist plays an essential role in this field because he must be trained to early detect the manifestations of the disease in the oral cavity, which would prevent its progression.

Materials and methods: A cross-sectional, descriptive study was conducted, with 20 patients who consulted three stomatology services in South America; a stomatological examination was done and an exhaustive clinical history was obtained; the lesions found were classified and the other data collected were analyzed.

Results: The lesion prevalent in primary syphilis was ulcers and only one case was detected; about the secondary form, nineteen cases were detected, and multiple associated lesions were found; finally, no case of tertiary syphilis was found.

Conclusions: Disclosure of these results and further research in this field are considered essential to educate and thus prevent the progression of syphilis to a latent or tertiary form that endangers the life of the population.
\end{abstract}

Keywords: sexually transmitted diseases, mouth neoplasms, serodiagnosis of syphilis, syphilis.

\section{Lesões bucais características da sífilis, encontradas em três serviços de estomatologia da América Latina}

\begin{abstract}
Resumo
Introdução: a sífilis é uma doença considerada como antiga, que tem causado grandes estragos durante toda a história da humanidade; apesar de métodos de proteção sexual e antibióticos para combater a infecção, na atualidade, ainda é preocupante sua prevalência. 0 odontologista desempenha um papel imprescindível nesse campo, devido a que deve ser capacitado para detectar as manifestações da doença em cavidade bucal de uma forma precoce, com o que se evitaria sua progressão.

Metodologia: realiza-se um estudo descritivo de corte transversal, com 20 pacientes que consultaram três serviços de estomatologia da América do Sul; é realizado exame estomatológico com eles e levantado seu prontuário médico.

Resultados: a lesão prevalente em sífilis primária foi a úlcera e somente foi detectado um caso; sobre a forma secundária, foram detectados 19 casos e encontradas múltiplas lesões associadas; por último, não se constatou nenhum caso de sífilis terciária.

Conclusões: consideram-se fundamentais a divulgação desses resultados e o desenvolvimento de pesquisas no campo a fim de educar os profissionais da saúde e, assim, evitar a progressão da doença a uma forma latente ou terciária que coloque em risco a vida da população.
\end{abstract}

Palavras-chave: doenças de transmissão sexual, neoplasias da boca, sorodiagnóstico da sífilis, sífilis. 


\section{Introducción}

La sífilis es una infección de transmisión sexual causada por el espiroqueta Treponema pallidum. La sífilis es transmisible por contacto sexual con lesiones infecciosas, de la madre al feto dentro del útero, a través de la transfusión de sangre y a veces por medio de roturas en la piel que entran en contacto con lesiones infecciosas. Si no se trata, progresa a través de cuatro etapas: primaria, secundaria, latente y terciaria $[1,2]$.

Treponema pallidum es una bacteria espiral frágil de 6-15 micrómetros de largo por 0,25 micrómetros de diámetro. Su pequeño tamaño la hace invisible en microscopía de luz; por lo tanto, debe ser identificada por sus movimientos ondulantes distintivos en la microscopía de campo oscuro. Solo puede sobrevivir brevemente fuera del cuerpo. Por lo tanto, la transmisión casi siempre requiere contacto directo con la lesión infecciosa [3].

T. pallidum se replica en el sitio de la inoculación inicial, dividiéndose una vez cada 30-33 horas, con lo que induce una respuesta inflamatoria local que resulta en un chancro indoloro aproximadamente 3-6 semanas después de la infección inicial. En cada chancro, las espiroquetas proliferantes están rodeadas por células inmunes, incluyendo células T CD 4 + y CD8 +, células plasmáticas y macrófagos, que producen citoquinas IL-2 e IFN- $\gamma$, indicando una respuesta Th1 $[4,5]$.

La necrosis de los tejidos y la ulceración ocurren debido a la vasculitis de los vasos pequeños, y el tráfico de células inmunes causa una linfadenopatía regional no sensible. En 3-8 semanas el chancro se cura, lo que indica la separación de T. pallidum localmente. Sin embargo, en este momento, dicha bacteria se ha propagado sistemáticamente a múltiples tejidos y órganos, preparando el escenario para la sífilis secundaria $[6,7]$.

T. pallidum se propulsa mediante un mecanismo tipo sacacorchos girando alrededor de su eje longitudinal, utilizando endoflagella contenida dentro del espacio periplásmico entre la membrana citoplasmática y la membrana externa $[8,9]$. Este atraviesa las uniones estrechas entre las células endoteliales para entrar en los espacios perivasculares, donde gran número de treponemas y células inmunes se acumulan. Con base en imágenes de microscopía electrónica de lesiones secundarias de la sífilis, T. pallidum también puede utilizar transcitosis para propagarse a través del endotelio.
Este puede inducir la producción de MMP-1, que degrada el colágeno y puede facilitar el acceso y la salida del torrente sanguíneo, dando lugar a la diseminación sistémica $[10,11]$.

Generalmente, en los tres meses siguientes a la infección, aparecen síntomas de sífilis secundaria. La manifestación clínica más común es una erupción maculopapular diseminada. Los síntomas adicionales pueden incluir malestar, pérdida de peso, dolores musculares, linfadenopatía generalizada, alopecia irregular, meningitis, inflamación ocular, parches mucosos (inflamación localizada de los tejidos mucosos de la cavidad oral y los genitales), hepatitis y dismotilidad gástrica $[12,13]$.

Tras la sífilis secundaria, el paciente entra en un periodo latente durante el cual el diagnóstico solo se puede hacer mediante pruebas serológicas y su transmisión sexual es rara, generalmente se transmite por vía intrauterina. Este periodo se divide, a su vez, en latente precoz y en latente tardío. La recaída de una sífilis secundaria es más probable en esta fase precoz y se produce como consecuencia de una disfunción inmunológica. La sífilis tardía o terciaria se refiere a la aparición de manifestaciones clínicas, aparentes o inaparentes, que se desarrollan en más de un tercio de los pacientes no tratados y cuya base patológica son las lesiones características denominadas "gomas", que se presentan sobre todo en piel y suelen destruir tejido causando comunicaciones entre cavidades; las oronasales no son frecuentes, pero son de importante interés para el odontólogo [14].

Existe otro tipo de sífilis que se transmite de madre a hijo en el útero o durante el parto, se denomina "sífilis congénita" y se divide en dos: la precoz y la tardía. La sífilis congénita precoz se manifiesta a los tres meses de edad o antes, y se caracteriza por erupciones y lesiones maculopapulosas en todo el cuerpo; a los ocho meses tiende a causar osteocondritis y seudoparálisis. La tardía se manifiesta después de los 2 años de edad y causa úlceras gomosas que tienden a comprometer la nariz, el tabique y el paladar duro, y lesiones periósticas que dan origen a las tibias en sable y al abovedamiento de los huesos frontal y parietal $[15,16]$.

Para el diagnóstico de dichas infecciones, hay varios tipos de pruebas de laboratorio clasificadas en dos grupos: las pruebas no treponémicas miden inmunoglobulinas IgG e IgM, que son producidas en los tejidos dañados por el treponema. Puesto que no miden anticuerpos específicos frente a 
T. pallidum, su positividad no asegura la infección sifilítica y se utilizan para evaluar la eficiencia del tratamiento; estas son: VDRL, RPR, TRUST, ELISA y USR. Por su parte, las pruebas treponémicas se utilizan principalmente para confirmar los resultados positivos obtenidos con las pruebas reagínicas, debido a que producen escasos o nulos falsos positivos; estas son: la FTA-ABS (más utilizada), la TPHA y la Western blot $[17,18]$.

Para el tratamiento de dicha infección, se debe aplicar antibioticoterapia lo más pronto posible. El antibiótico de elección es la penicilina G IM. En pacientes alérgicos en fase primaria y secundaria, se recomienda tratar con doxiciclina vía oral 100 mg por 21 días, y en fase tardía o terciaria es recomendable desensibilización para tratar con penicilina [19].

A escala internacional, la prevalencia de la sífilis varía según la región. La sífilis sigue siendo frecuente en muchos países en desarrollo y en algunas zonas de América del Norte, Asia y Europa, especialmente en Europa oriental. Las tasas más altas se dan en el sur y sureste de Asia, seguido de cerca por África. Las terceras tasas más altas se encuentran en las regiones de América Latina y el Caribe. La mayoría de los casos nuevos ocurren en hombres y mujeres de 20 a 29 años [20].

Debido a lo anterior, es necesario que el odontólogo tenga la capacidad de diagnosticar esta infección en sus estadios iniciales y así evitar su progresión a una fase sistémica, en la cual comienza a tener graves repercusiones en todo el organismo e incluso puede llevar a la muerte [21]. Según Grinspan [22], el examen estomatológico exhaustivo debe seguir esta secuencia sistemática: examen extraoral (ATM, cadenas ganglionares) zona perioral, semimucusa labial, mucosa yugal, paladar duro, paladar blando, orofaringe, cara dorsal de lengua, bordes laterales de lengua, cara ventral de lengua, piso de boca, encía y dientes. Con dicho protocolo, se evitará pasar por alto lesiones en cavidad bucal.

\section{Metodología}

Se realiza un estudio descriptivo de corte transversal. Participaron veinte pacientes que acudieron a los servicios de estomatología de las facultades de Odontología de las Universidades de Buenos Aires y Cartagena y del Hospital Alemán en Buenos
Aires. Previa calibración del clínico, al total de la muestra se les realiza historia clínica exhaustiva incluyendo examen estomatológico minucioso (secuencia según Grinspan), además de pruebas de VDRL y FTA-ABS para el diagnóstico. Se realiza finalmente análisis de datos.

\section{Resultados}

El promedio de edad de los veinte pacientes fue de 35 años, el de menor edad tiene 18 años y el de mayor edad tiene 69 años, ocho (es decir, el 40\%) son mujeres y doce (es decir, el 60\%) son hombres. Acerca de sus hábitos y vicios, diez pacientes (es decir, el 50\%) son fumadores y solo una persona (es decir, el 5\%) es consumidor de alcohol frecuente (Tabla1).

Tabla 1. Descripción de hábito/vicios de la población de estudio

\begin{tabular}{lcc}
\hline Hábito/vicio & $\begin{array}{c}\text { Número de } \\
\text { personas }\end{array}$ & Porcentaje \\
\hline Tabaquismo & 10 & $50 \%$ \\
Alcoholismo & 1 & $5 \%$ \\
No presenta & 9 & $45 \%$ \\
Totales & 20 & $100 \%$ \\
\hline
\end{tabular}

Fuente: elaboración propia

De estos veinte pacientes, diecinueve (es decir, el 95\%) fueron diagnosticados con sífilis secundaria, solo un paciente (es decir, el 5\%) fue diagnosticado con sífilis primaria y no se encontró ningún caso de sífilis terciaria (Tabla 2).

Tabla 2. Descripción de diagnósticos de sífilis en sus diferentes etapas

\begin{tabular}{lcc}
\hline Subtipo de sífilis & $\begin{array}{c}\text { Número de } \\
\text { personas }\end{array}$ & Porcentaje \\
\hline Secundaria & 19 & $95 \%$ \\
Primaria & 1 & $5 \%$ \\
Terciaria & 0 & $0 \%$ \\
Totales & 20 & $100 \%$ \\
\hline
\end{tabular}

Fuente: elaboración propia 
En los pacientes diagnosticados con sífilis secundaria, predominó la pápula en un $57,8 \%$, luego la úlcera en un $36,8 \%$ y la queratosis en un $5,2 \%$ (Tabla 3 y Figuras 1-6). Solo un paciente fue diagnosticado con sífilis primaria y la lesión encontrada fue la úlcera.

Tabla 3. Descripción de lesiones encontradas de la sífilis secundaria

\begin{tabular}{lcc}
\hline $\begin{array}{c}\text { Tipo de lesión de } \\
\text { sífilis secundaria }\end{array}$ & $\begin{array}{c}\text { Número de } \\
\text { personas }\end{array}$ & Porcentaje \\
\hline Pápula & 11 & $57,8 \%$ \\
Úlcera & 7 & $36,8 \%$ \\
Queratosis & 1 & $5,2 \%$ \\
Totales & 19 & $100 \%$ \\
\hline
\end{tabular}

Fuente: elaboración propia

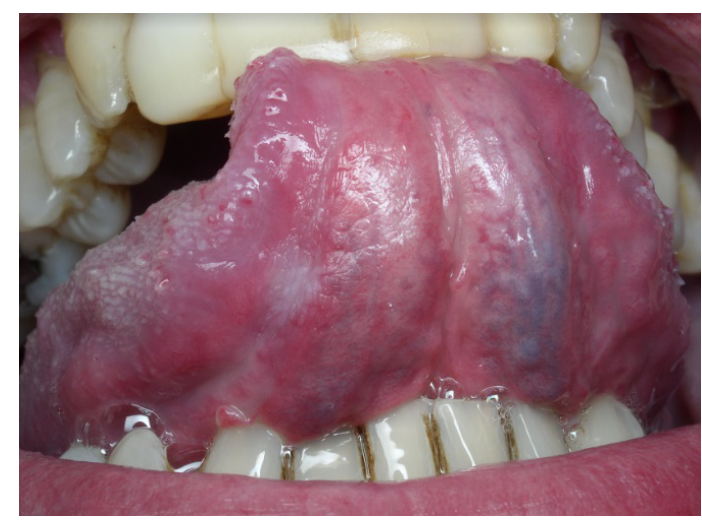

Figura 1. Fotografía frontal intraoral, se evidencia queratosis en cara ventral de lengua

Fuente: elaboración propia

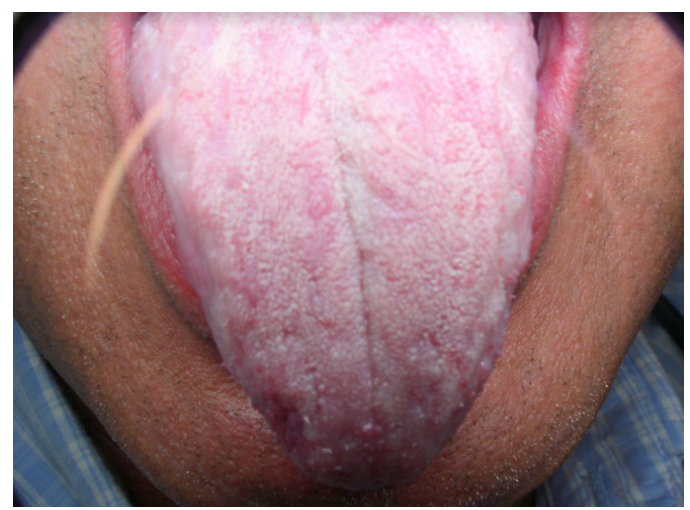

Figura 2. Fotografía frontal intraoral, se evidencian múltiples pápulas en cara dorsal de lengua

Fuente: elaboración propia

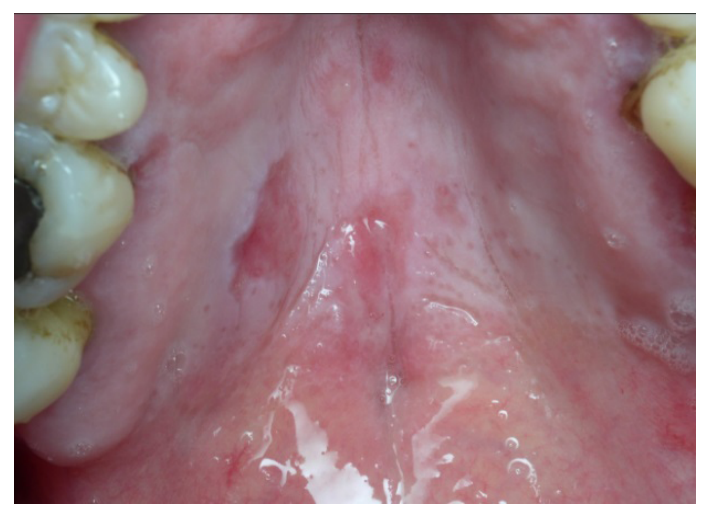

Figura 3. Fotografía intraoral con espejo bucal, se evidencian úlceras en paladar duro a nivel de primeros molares Fuente: elaboración propia

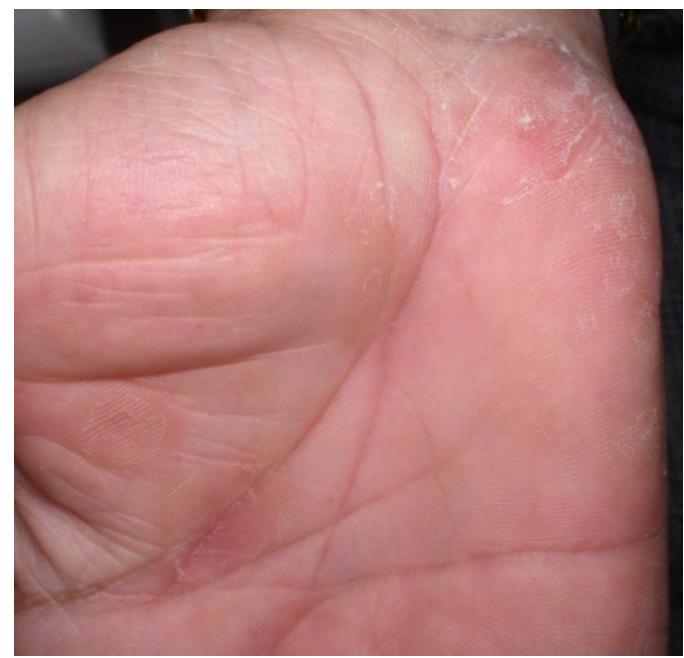

Figura 4. Se evidencian lesiones descamativas en palma de mano características de sífilis secundaria Fuente: elaboración propia

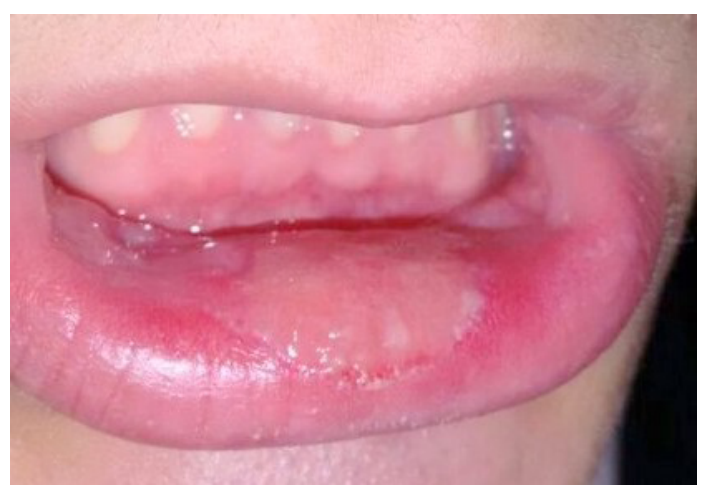

Figura 5. Fotografía extraoral frontal, se evidencia chancro sifilítico en mucosa labial inferior Fuente: elaboración propia 


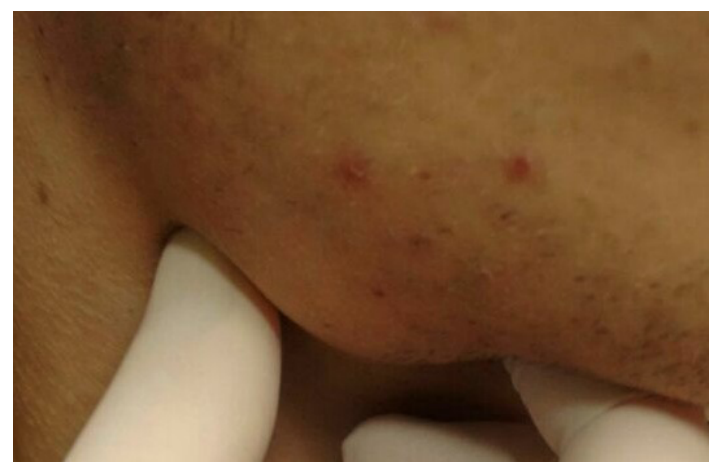

Figura 6. Ganglio satélite de la cadena submandibular Fuente: elaboración propia

El sitio anatómico en cavidad bucal en el que se encontró más afectación en la población de estudio fue la mucosa yugal, alterada en nueve personas (es decir, en el 18\%), seguido por el paladar blando y la cara dorsal de lengua, alterados en ocho personas cada uno (es decir, en el 16\% para cada uno). Luego en la escala se encuentra la mucosa labial, alterada en siete personas (es decir, en el $14 \%) ; ~ l u e g o$ siguen los bordes laterales de lengua, alterados en seis personas (es decir, en el 12\%); y por último se encuentra la encía, la cara ventral de lengua y el piso de boca, que se hallaron afectados en cuatro personas cada uno (es decir, en el $8 \%$ para cada uno; Tabla 4).

Tabla 4. Descripción de prevalencia de afectación de sitios anatómicos en cavidad bucal

\begin{tabular}{lcc}
\hline \multicolumn{1}{c}{ Sitio anatómico } & $\begin{array}{c}\text { Número de } \\
\text { personas }\end{array}$ & Porcentaje \\
\hline Mucosa yugal & 9 & $18 \%$ \\
Paladar blando & 8 & $16 \%$ \\
Cara dorsal de lengua & 8 & $16 \%$ \\
Mucosa labial & 7 & $14 \%$ \\
Bordes laterales de lengua & 6 & $12 \%$ \\
Encía & 4 & $8 \%$ \\
Cara ventral de lengua & 4 & $8 \%$ \\
Piso de boca & 4 & $8 \%$ \\
\hline
\end{tabular}

Fuente: elaboración propia

De los veinte sujetos estudiados, solo dos (es decir, el 10\%) fueron diagnosticados con ViH positivo, ambos padecían sífilis secundaria (Tabla 5).
Tabla 5. Descripción de diagnósticos de prueba de viH

\begin{tabular}{lcc}
\hline $\begin{array}{c}\text { Resultado } \\
\text { prueba viH }\end{array}$ & $\begin{array}{c}\text { Número de } \\
\text { personas }\end{array}$ & Porcentaje \\
\hline Positivo & 2 & $10 \%$ \\
Negativo & 18 & $90 \%$ \\
Totales & 20 & $100 \%$ \\
\hline
\end{tabular}

Fuente: elaboración propia

\section{Discusión}

La literatura es escasa acerca de las manifestaciones en cavidad bucal de la sífilis; a pesar de ser una infección muy antigua, los estudios en este campo son muy pocos.

Aguayo et al. [23] reportan un caso de sífilis en su forma primaria; el paciente acude a los servicios de urgencias por un nódulo de consistencia dura, asintomático, con superficie erosionada cubierta de costra serosa, localizado en el labio superior; en la anamnesis el paciente reconoció haber realizado prácticas sexuales orales; se hicieron pruebas de VDRL y biopsia escinsional que resultaron positivas para sífilis y se diagnosticó chancro sifilítico. En nuestro estudio no se encontró ninguna lesión en forma de nódulo, la lesión encontrada fue la úlcera en el $100 \%$ de los pacientes con sífilis primaria; además, cabe resaltar que las pruebas de VDRL fueron realizadas en el presente estudio, pero en el estudio de Aguayo et al. no se hizo la prueba FTA-ABS, que es necesaria para un diagnóstico sin falsos positivos.

Castro y Guzmán [24] describen un caso clínico de sífilis secundaria; el paciente presenta lesiones placoulcerativas dolorosas, que involucran paladar blando, labio superior e inferior; además, presenta lesiones descamativas y placas eritematosas en las palmas de las manos; se realiza prueba de VDRL que resulta positiva. Las lesiones encontradas en dicho estudio también fueron reportadas en el presente; sin embargo, dicho estudio no reporta lesiones papulares, que fueron encontradas en gran porcentaje en la presente investigación.

Bezerra et al. [25] reportan un caso de sífilis secundaria con lesiones atípicas; eran lesiones vesiculares que generaban prurito intenso. Estas lesiones no se encuentran en esta investigación, y la literatura reporta muy pocos casos de sífilis asociados. 
Un punto muy importante en los resultados de esta investigación fue encontrar la queratosis como lesión de sífilis secundaria, debido a que es muy infrecuente; de hecho, no hay reportes en la literatura al respecto. La queratosis sifilítica se considera un factor predisponente para el desarrollo de cáncer bucal [26], por lo cual estudios en este campo se consideran pertinentes.

En cuanto a la sífilis terciaria, nuestro estudio coincide con la literatura actual, debido a que no existen casos reportados acerca de esta forma de la sífilis que afecte tejidos o sitios anatómicos del sistema estomatognático. Los pocos estudios existentes la asocian a lesiones en piel [27].

Por último, se considera pertinente enfatizar la relación de la sífilis con el viH/sida. Diversos estudios [28-30] reportan que existe una alta prevalencia de pacientes infectados con ambas patologías, de aproximadamente 40 a 50\%; sin embargo, en el presente estudio solo el $10 \%$ de la población padecía de ambas. Aunque no hay coincidencia, no se le resta importancia a dicho dato, debido a que los pacientes diagnosticados con alguna de estas enfermedades deben recibir mayor educación para reducir el riesgo de una nueva infección.

\section{Conclusiones}

Se puede concluir que a pesar de que la sífilis es una infección bastante antigua, su prevalencia alrededor del mundo todavía se considera preocupante. Al conocer la patogenia, es importante saber identificar las lesiones antes descritas para detectar la infección en cavidad bucal antes de una progresión sistémica que afecte la calidad de vida del individuo o incluso la ponga en riesgo; se considera importante hacer más estudios en este campo, con una muestra mucho mayor, y divulgar estos resultados a la comunidad científica mundial.

\section{Referencias}

[1] Cuerda-Galindo E, Sierra-Valenti X, GonzálezLópez E, López-Muñoz F. Syphilis and human experimentation from World War II to the present: A historical perspective and reflections on ethics. Actas Dermosifiliogr. 2014;105(9):847-53. doi: http:// dx.doi.org/10. 1016/j.ad.2013.09.007.
[2] Berdasquera D, Lazo M, Galindo B, Gala A. Sífilis: pasado y presente. Rev Cubana Hig Epidemiol. 2004;42(2).

[3] Fraser CM, Norris SJ, Weinstock GM, White O, Sutton GG, Dodson R, et al. Complete genome sequence of Treponema pallidum, the syphilis spirochete. Science. 1998;281(5375):375-88.

[4] Cumberland MC, Turner TB. The rate of multiplication of Treponema pallidum in normal and immune rabbits. Am J Syph Gonorrhea Vener Dis. 1949;33(3):201-12.

[5] Fieldsteel AH, Cox DL, Moeckli RA. Cultivation of virulent Treponema pallidum in tissue culture. Infect Immun. 1981;32(2):908-15.

[6] Bjerke JR, Krogh HK, Matre R. In situ identification of mononuclear cells in cutaneous infiltrates in discoid lupus erythematosus, sarcoidosis and secondary syphilis. Acta Derm Venereol. 1981;61(5):371-80.

[7] Leader BT, Godornes C, Van Voorhis WC, Lukehart SA. CD4+ lymphocytes and gamma interferon predominate in local immune responses in early experimental syphilis. Infect Immun. 2007;75(6):3021-6. doi: http://dx.doi.org/10.1128/IAI.01973-06.

[8] Izard J, Renken C, Hsieh CE, Desrosiers DC, Dunham-Ems S, La VC, et al. Cryo-electron tomography elucidates the molecular architecture of Treponema pallidum, the syphilis spirochete. J Bacteriol. 2009; 191(24):7566-80. doi: http://dx.doi.org/10.1128/JB. 01031-09.

[9] Liu J, Howell JK, Bradley SD, Zheng Y, Zhou ZH, Norris SJ. Cellular architecture of Treponema pallidum: Novel flagellum, periplasmic cone, and cell envelope as revealed by cryo-electron tomography. J Mol Biol. 2010;403(4):546-61. doi: http://dx.doi. org/10.1016/j.jmb.2010.09.020

[10] Thomas DD, Navab M, Haake DA, Fogelman AM, Miller JN, Lovett MA. Treponema pallidum invades intercellular junctions of endothelial cell monolayers. Proc Natl Acad Sci USA. 1988;85(10):3608-12.

[11] Chung KY, Kim KS, Lee MG, Chang NS, Lee JB. Treponema pallidum induces up-regulation of interstitial collagenase in human dermal fibroblasts. Acta Derm Venereol. 2002;82(3):174-8.

[12] Chapel TA. The signs and symptoms of secondary syphilis. Sex Transm Dis. 1980;7(4):161-4.

[13] Mindel A, Tovey SJ, Timmins DJ, Williams P. Primary and secondary syphilis, 20 years' experience. 2. Clinical features. Genitourin Med. 1989;65(1):1-3.

[14] Ho E, Lukehart S. Syphilis: Using modern approaches to understand an old disease. J Clin Invest. 2011;121(12):4584-92. doi: http://dx.doi.org/ 10.1172/JCI57173. 
[15] Lugones M, Molinet I, Quintana T, Vázquez M. Sífilis y gonorrea; parte de su historia. Rev Cubana Med Gen Integr. 1995;11(4):382-4.

[16] Lazarini FM, Barbosa DA. Educational intervention in Primary Care for the prevention of congenital syphilis. Rev Lat Am Enfermagem. 2017;25:e2845. doi: http://dx.doi.org/10.1590/1518-8345.1612.2845.

[17] Romero M, Suárez I, Fajardo J, Barón B. Sífilis maligna en el paciente infectado por el virus de la inmunodeficiencia humana $(\mathrm{VIH})$ : descripción de un caso y revisión de la literatura. An Med Interna. 2003;20(7):45-8.

[18] Morales-Múnera CE, Fuentes-Finkelstein PA, Vall Mayans M. Update on the diagnosis and treatment of syphilis. Actas Dermosifiliogr. 2015;106(1):68-9. doi: http://dx.doi.org/10.1016/j.ad.2014.06.007.

[19] Riedner G, Rusizoka M, Todd J, Maboko L, Hoelscher M, Mmbando D, et al. Single-dose azithromycin versus penicillin $G$ benzathine for the treatment of early syphilis. N Engl J Med. 2005;353 (12):1236-44. doi: http://dx.doi.org/10.1056/NEJM oa044284.

[20] World Health Organization (wHo). Global prevalence and incidence of selected curable sexually transmitted infections: Overview and estimates. Geneva: wHo; 2001.

[21] Villar R, Porta J. Neurosyphilis in the 21st century: A descriptive study in a tertiary hospital in Madrid. Rev Neurol. 2016;63(9):393-402.

[22] Grinspan D. Enfermedades de la boca: semiología, patología, clínica y terapéutica de la mucosa bucal. Tomo 1. Buenos Aires: Mundi; 1970.

[23] Aguayo I, Moreno C, Vano S, Jaen P. Chancro sifilítico en el labio: una localización poco frecuente. Cartas científicas / Enferm Infecc Microbiol Clin. 2010;28(2):134-40.
[24] Castro S, Guzmán D. Manifestaciones bucales por sífilis secundaria. Reporte de un caso clínico. Rev Cient Odontol. 2015;11(1).

[25] Duarte Bezerra Pinto R, Rubim Bellott Batista Nascimento T, Pacheco Bicalho de Andrade J, Policarpo F, Ribeiro Estrella R, Guedes Vilar E. Lesões vesiculares e prurido intenso em paciente com sífilis secundária: manifestação clínica atípica. Dermatol Online J. 2016;22(6). pii: 13030/qt91h7g8k7.

[26] Meza G, Muñoz J, Páez C, Cruz B, Aldape B. Carcinoma de células escamosas de cavidad bucal en un centro de tercer nivel de atención social en la Ciudad de México: experiencia de cinco años. Av Odontoestomatol. 2009;25(1):19-28.

[27] Garritano M, Jaurigoity M, Maradeo M, Rafti P, Corrons F. Sífilis terciaria cutánea: una gran desconocida. Presentación de un caso y revisión bibliográfica. Arch Argent Dermatol. 2008;58:97-103.

[28] González C, Antequera I, Clavijo E, Márquez M, Santos J, Palacios R. Syphilis and human immunodeficiency virus infection: An endemic infection in men who have sex with men. Enferm Infecc Microbiol Clin. 2015;33(1):32-6. doi. http://dx.doi.org/10. 1016/j.eimc.2014.07.013.

[29] Gallego-Lezáun C, Arrizabalaga Asenjo M, González-Moreno J, Ferullo I, Teslev A, Fernández-Vaca $\mathrm{V}$, et al. Syphilis in men who have sex with men: A warning sign for HIV infection. Actas Dermosifiliogr. 2015;106(9):740-5. doi: http://dx.doi.org/10.1016/j. ad.2015.05.010.

[30] Lopes L, Ferro R, Llobet S, Lito L, Borges J. Syphilis: Prevalence in a hospital in Lisbo. Acta Med Port. 2016;29(1):52-5. doi: http://dx.doi.org/10.20344/am p.6247. 